\title{
Challenges for developing high temperature superconducting ring magnets for rotating electric machine applications in future electric aircrafts
}

\author{
Mohammad Yazdani-Asrami, Min Zhang, and Weijia Yuan \\ Applied Superconductivity Laboratory, Department of Electronic and Electrical Engineering, University of \\ Strathclyde, Glasgow, United Kingdom \\ Email: m.yazdaniasrami@gmail.com
}

\begin{abstract}
:
One of the most promising applications for high temperature superconducting (HTS) material is magnet manufacturing. HTS magnets are considered to be used in wide range of applications such as MRIs, particle accelerators, as well as electrical machines. HTS rotating machines are mainly utilized for cryo-electrification, i.e. application of superconductivity for modern electrification. One of the main aspects of cryo-electrification is electric aircraft application which is recently enabled by continuous progress in design development of superconducting magnets and HTS machines.

This paper discusses the challenges in front of application of a newly developed magnet type, i.e. HTS ring magnets for being used in superconducting rotating machine in future electric aircrafts. HTS ring magnet is compact, easy to develop, fault tolerant, and light weight which recently reached to a high level of magnetic field.
\end{abstract}

Keywords: Electric Aircraft, HTS ring magnet, Superconducting machine

\section{Introduction:}

High temperature superconducting (HTS) materials in the form of coated conductor tapes attracted a huge attention for electrical machine applications by the beginning of $21^{\text {st }}$ century. Application of superconducting electric machines are recently in the center of attention because it is the radical technology solution for enabling the idea of full electric aircraft [1-3]. Utilizing superconducting motors and generators compared to their traditional counterparts lead to higher power and torque densities. There are some reasons for this, including [4-6]:

1) The current carrying capacity of HTS tapes are much higher than other superconductors and traditional conductors, e.g. the typical current density of copper winding in electric machines is 4 to $6 \mathrm{~A} / \mathrm{mm}^{2}$, whilst this number is about 100 to 500 times higher (depends on the tape material and manufacturer) for superconducting machines made out of $2 \mathrm{G} \mathrm{HTS}$ tapes at $77 \mathrm{~K}$ to $20 \mathrm{~K}$, temperature of LN2 to LH2. Note that recently the idea of full electric aircraft looks achievable more than before by having the hydrogenbased system with fuel cell instead than battery and cryocoolers, for energy storage and cooling purposes.

2) The great capability of HTS tapes to produce higher magnetic fields in magnets, e.g. the magnetic loading in traditional machines are about $1 \mathrm{~T}$ and normally produced by rare-earth permanent magnets, whilst using 2G HTS tapes, this value can easily be lifted to 3 to 4 T. Therefore, this high level of magnetic loading will enable the idea of coreless superconducting machine configuration which further leads to higher power and torque densities as well as lighter weight machines.

3) Lower AC loss of HTS tapes compared to conventional conductors. It is very important for electrical machine to increase their efficiency.

Therefore, by using HTS superconductors reaching the power density target of $20 \mathrm{~kW} / \mathrm{Kg}$ for electrical machines in electric aircraft seems to be achievable [6-7].

There are quite reasonable number of publications in literature focused on increasing the electrical loading of superconducting machines by using HTS materials [1-2]. They achieved much higher current density by simply replacing copper and aluminium conductors with $2 \mathrm{G} \mathrm{HTS}$ tapes. But, increasing the magnetic loading 
in machine seem to be more challenging, since not only one needs to deal with the extra core and conductor losses (including AC loss) caused by higher field (which cooling system should be able cope with that) but also the critical current of tapes in armature would be significantly reduced which needs to be considered during the design stage. There are different topologies for field production/generation part of these machines. These topologies are including traditional permanent magnet, bulk superconductors, and stacked tapes [8]. It is technically very difficult to achieve $20 \mathrm{~kW} / \mathrm{Kg}$ power density target without increasing the magnetic loading of superconducting electric machines. Superconducting trapped-field magnets, by trapping persistent currents are a promising solution for increasing the magnetic loading in HTS electric machines.

There are some publications in literature on developing HTS bulk magnets with high trap fields up to $17.7 \mathrm{~T}$ [9-11]. But the main problems with bulk magnets are that they are quite mechanically vulnerable in larger size magnets and cracks may appear in their construction at low temperatures, which makes them not a safe and reliable option for field magnet of high-power superconducting machines for electric aircraft applications, where safety is a crucial priority. The perspective of target speeds for these superconducting machines are above $20000 \mathrm{rpm}$. At this speed, any cracks on the surface of magnet could lead to a quite severe damage to magnet itself, and eventually to machine construction. In addition, temperature should be below $20 \mathrm{~K}$ for having much higher field bulk magnet. On the other hand, there are issues for stacked type magnets including difficulty for producing larger size magnets above $50 \mathrm{~mm}$ because of the existing technical limitations in tape manufacturing. The solution for these challenges seems to be HTS ring magnets. This type of magnet was developed based on the jointless persistent current idea by milling a slit along the tape length, so that the HTS film forms a closed path for supercurrent [12-13]. By stacking the split tapes together, higher magnetic field can be achieved. In [14], 4.6 T as the highest trapped field in the world was achieved in an HTS ring magnet in Applied Superconductivity Laboratory of University of Strathclyde, Glasgow, United Kingdom, by HTS persistent current loop using field cooling magnetization method at $25 \mathrm{~K}$. This absolutely exciting magnetic field achievement open a new route for research to use such a powerful and compact magnet in rotating electric machines especially for future electric aircraft applications.

\section{Challenges in front of application of ring magnets}

The following issues will need to be considered and addressed for utilizing HTS ring magnet in superconducting electric machines for electric aircrafts:

1) Mechanical strength of the HTS ring magnet is highly important for the rotating electric machine applications. It is related to machine speed as well as magnet shape. In electric aircraft, speed of the motor would be in the range of 16000 to $24000 \mathrm{rpm}$ or sometimes even higher, therefore mechanical strength of the ring magnet is still a concern, even though this type of magnet performs much better and more reliable than bulk magnets. On the other hand, size and shape of magnets demand a shorter or longer slit, and therefore, stress and strain applied to tapes need to be considered during design stage.

2) Size and weight of a superconducting motor in electric aircraft is highly critical from power density point of view. Therefore, using a magnet with proper size would be of great interests. This HTS ring magnet can be feasibly and flexibly designed and assembled compared to other types of superconducting magnets. By changing the length of the slit along the tape length or stack the magnets on top of each other, the diameter or height of the assembled magnet would be adjusted. On the other hand, one can optimize the shape, size, and resultant magnetic field of the HTS ring magnet using artificial intelligence and machine learning methods through running an optimization problem. For this purpose, softwares such as COMSOL Multiphysics could be linked with artificial intelligence-based or machine learning algorithms to find the optimized design, shape, size, number of tapes, and opening angles in the magnet construction. For solving the problem, field strength level as well as its homogeneity and uniformity could be defined as objective functions. In rotating machine applications, it is highly important to have an optimized magnet design to increase the magnetic loading of 
machine and consequently its torque density (torque to mass) and power density (power to mass). Note that using machine learning methods would be highly desirable, since one can use this approach with additive manufacturing in order to reach to a more client oriented and in the end machine-oriented design.

3) Field uniformity is important in electric machine applications because distribution and uniformity of magnetic field will affect the magnetic loading, power/torque density, and thermal behavior of machine. One way to have a higher field uniformity is to optimize the ring design by adding multiple smaller ring magnets into a larger one. This will further correct any nonuniformity and will increase the current density of tape or in a better explanation will increase the utilization factor of the critical current in them. But to have highest field homogeneity, several smaller magnets may be needed, which the number of these smaller magnets should be determined by a trade-off between cost, size, number of tapes, and the field homogeneity constraints for the application. In other words, new stacking methods need to be studied to enhance the magnetic field homogenization, considering construction, parameters, and specifications of the rotor or (sometimes stator) of practical rotating machine in future electric aircraft applications.

4) Effect of epoxy materials on magnet performance from mechanical stability, critical current degradation, and cooling viewpoints need to be studied. Some epoxy resins such as Stycast, and Polyurethane need to be used to immobilize the stacks and increase the mechanical stability of ring magnet in high rotational speed of the machine. One needs to consider effect of epoxy material on the transversal and longitudinal stress on the surface of tapes. In other words, transverse tensile stresses are a problem when an epoxy resin is used to impregnate and stabilize the tapes in the ring magnet [15-16]. Epoxy may degrade the critical current of the tapes as time goes by, and the magnet performance (including magnitude of magnetic field and uniformity of field) will degrade, consequently. It will have a dramatic impact on rotating machine performance especially when it is going to be used in such a critical application like electric aircraft. The output torque/power of machine depends on the magnitude of the magnetic field density produced by magnet. On the other hand, the field uniformity impacts loss generation, and as a consequence temperature distribution in the machine windings. In addition, nonuniform distribution of magnetic field will cause eccentricity faults in machine which further damage the shaft, wear the bearings, and increase the vibration and noise in machine. On the other hand, the type of epoxy will affect the cooling process, and it would be better to use epoxies with high thermal conductivity and low thermal expansions. In addition, effect of thermal cycling on composite epoxies need to be experimentally tested. Since, epoxy may lose part of its stability after some cooling transients or cycles.

5) Effective cooling for magnets is essential to reach the best performance. Because it will increase current density in tapes and as a result will increase the magnitude of resultant magnetic field. Using epoxies with higher thermal conductivity would be useful. In addition, some powder fillers could be added to the epoxy to increase conduction cooling performance. The best existing filler seems to be Beryllium oxide or so-called Beryllia; another cheaper and weaker options would be Alumina. This will not only increase the heat transfer for effective cooling but also provide a uniform temperature distribution. The temperature uniformity would be more important for larger magnets with higher field, or lower field magnets but with compact design. Any hotspots or critical current weak-points lead to an uneven temperature and field distributions. Effect of these powder fillers on critical current degradation should be studied and examined before fabricating the magnet for electric machines, during R\&D stages.

6) Using this type of ring magnets is highly favorable for electric machine applications, together with a charging system, which is missing at the moment. A magnetizing flux pump will need to be developed for ring magnets in the electric machine applications. Recently, there is promising development on flux pump technology.

7) Fault tolerance capability of this type of ring magnets are superior since each loop is carrying the persistent current independently, thus, a fault in an individual loop would not end up with magnet failure. But, a major 
fault in internal layers will change the temperature distribution, current density, and magnetic flux density of whole magnet assembly. Therefore, still there is a reasonable need for developing smart condition monitoring technique for fault detection of this type of magnets.

\section{Conclusion}

In this paper, challenges of design development and manufacturing of HTS ring magnets for being used in rotating machine of future electric aircraft were discussed. Magnet optimal design, mechanical stability, epoxy resin performance, cooling issues, flux pumping, and fault related issues were introduced, explained, and discussed as main challenges.

\section{References:}

[1] K. S. Haran, S. Kalsi, T. Arndt, H. Karmaker, R. Badcock, B. Buckley, T. Haugan, M. Izumi, D. Loder, J. W. Bray, P. Masson, and E. W. Stautner, "High power density superconducting machines - development status and technology roadmap," Supercond. Sci. Technol., 30, 2017, 1-30.

[2] T. Balachandran, D. Lee, N. Salk, K. S. Haran, A fully superconducting air-core machine for aircraft propulsion, IOP Conference Series: Materials Science and Engineering, 756, 2020, 012030.

[3] P. N. Barnes, M. D. Sumption, and G. L. Rhoads, Review of high power density superconducting generators: Present state and prospects for incorporating YBCO windings, Cryogenics, 45, 2005, 670-686.

[4] F. Weng, M. Zhang, T. Lan, Y. Wang, and W. Yuan, Fully superconducting machine for electric aircraft propulsion: study of AC loss for HTS stator, Supercond. Sci. Technol., 33, 2020, 104002.

[5] A. M. Campbell, Superconducting and conventional machines, Supercond. Sci. Technol., 27, 2014, 124012.

[6] M. Feddersen, K. S. Haran, and F. Berg, AC Loss Analysis of MgB2-Based Fully Superconducting Machines, IOP Conf. Series: Materials Science and Engineering, 279, 2017, 012026.

[7] M. Filipenko, L. Kühn, T. Gleixner, M. Thummet, M. Lessmann, D. Möller, M. Böhm, A. Schröter, K. Häse, J. Grundmann, M. Wilke, M. Frank, P. van Hasselt, J. Richter, M. Herranz-Garcia, C. Weidermann, A. Spangolo, and M. Klöpzig, Concept design of a high power superconducting generator for future hybrid-electric aircraft, Supercond. Sci. Technol., 33, 2020, 054002.

[8] Y. Terao, Y. Ishida, and H. Ohsaki, Electromagnetic Characteristic Comparison of Superconducting Synchronous Motor Characteristics for Electric Aircraft Propulsion Systems, SAE Technical Paper, 2019-01-1912, 2019.

[9] D. Zhou, M. Izumi, M. Miki, B. Felder, T. Ida, and M. Kitano, An overview of rotating machine systems with high temperature bulk superconductors, Supercond. Sci. Technol., 25, 2012, 103001.

[10] J. H. Durrell, A. R. Dennis, J. Jaroszynski, M. D. Ainslie, K. G. B. Palmer, Y. H. Shi, A. M. Campbell, J. Hull, M. Strasik, and E. E. Hellstrom, A trapped field of $17.6 \mathrm{~T}$ in melt processed bulk $\mathrm{Gd}-\mathrm{Ba}-\mathrm{Cu}-\mathrm{O}$ reinforced with shrink-fit steel, Supercond. Sci. Technol., 27, 2014, 082001.

[11] A. Patel, A. Baskys, T. Mitchell-Williams, A. McCaul, W. Coniglio, J. Hanisch, M. Lao, and B. A. Glowacki, A trapped field of 17.7 T in a stack of high temperature superconducting tape, Supercond. Sci. Technol., 31, 2018, 09 LT01.

[12] H. G. Lee, J. G. Kim, S. W. Lee, W. S. Kim, S. W. Lee, K. D. Choi, G. W. Hong, and T. K. Ko, Design and fabrication of permanent mode magnet by using coated conductor, Physica C: Superconductivity and its Applications, 445-448, 2006, 1099-1102.

[13] G. A. Levin, P. N. Barnes, J. Murphy, L. Brunke, J. D. Long, J. Horwath, and Z. Turgut, Persistent current in coils made out of second generation high temperature superconductor, wire Appl. Phys. Lett., 93, 2008, 062504.

[14] M. Z. Ali, J. Zheng, F. Huber, Z. Zhang, W. Yuan, and M. Zhang, 4.6 T generated by a high-temperature superconducting ring magnet, Supercond. Sci. Technol., 33, 2020, 04 LT01.

[15] C. Barth, N. Bagrets, K. P. Weiss, C. M. Bayer, and T. Bast, Degradation free epoxy impregnation of REBCO coils and cables, Supercond. Sci. Technol., 26, 2013, 055007.

[16] D. Yu, Y. Sun, H. Zhang, Y. Meng, and H. Liu, Experimental Analysis of Critical Current and Alternating Current Losses of High-Temperature Superconductor Tape with Resin and Gallium-Indium-Tin, Materials, 11, 2018, 573. 